\title{
PREVALÊNCIA DE SOBREPESO E OBESIDADE EM ADOLESCENTES MASCULINOS NAS MACRORREGIÕES DO BRASIL, 1980 - 2000
}

\author{
Prevalence of Overweight and Obesity in Male Adolescents \\ from Five Regions of Brazil, 1980-2000 \\ Predominio del Sobrepeso y Obesidad en Adolescentes Masculinos \\ de Cinco Regiones del Brasil, 1980-2000
}

Vera Lucia de Vasconcelos

Tiago Maria Lapa

Eduardo Freese de Carvalho

\begin{abstract}
Resumo
0 objetivo deste estudo foi determinar a prevalência e a tendência temporal do sobrepeso/obesidade em adolescentes brasileiros do sexo masculino. Métodos: Utilizou-se como fonte de informações o Banco de Dados do Exército Brasileiro. Esses dados foram coletados entre 1980 e 2000 e consta de 4.031.297 adolescentes entre 17 e 19 anos de idade. Foram realizados cinco cortes transversais, e quantificou-se a prevalência do sobrepeso/obesidade. Considerou-se sobrepeso 0 índice de massa corporal (IMC) entre 25 e $29,9 \mathrm{Kg} / \mathrm{m}^{2}$, e obesidade, IMC $>30 \mathrm{Kg} / \mathrm{m}^{2}\left(\mathrm{OMS}^{26}\right)$. Em todos os estados brasileiros, a prevalência de sobrepeso foi maior que a de obesidade, apesar de a velocidade de incremento ter sido mais alta para obesidade. Ao ser comparada a prevalência do início (1980) com a do final do estudo (2000), observou-se, no Brasil, uma razão de prevalência de 2,58 para sobrepeso e de 8,18 para obesidade. As prevalências de sobrepeso/ obesidade mostraram curvas ascensionais com tendência temporal crescente. As informações obtidas neste estudo apontam a necessidade de ações concretas para a prevenção de doenças crônicas em adultos jovens brasileiros.
\end{abstract}

Palavras-chave: Sobrepeso. Obesidade. Transição Nutricional. Epidemiologia. Saúde do Adolescente.

\begin{abstract}
The objective of this study was to determine the prevalence and the secular trend of overweight / obesity in adolescents Brazilians of the masculine sex. Methods A Brazilian Army's data bank. Those data were collected among 1980 to 2000, and it consists of 4.031.297 adolescents between 17 and 19 years of age. A cross-sectional study, with five cuts, was performed, and the prevalence of overweight and obesity was quantified. Overweight was considered when the Body Mass Index (BMI) ${ }^{3}$ reached between 25 and $29.9 \mathrm{Kg} / \mathrm{m}^{2}$ and obesity when the BMI was $>30 \mathrm{Kg} /$ $\mathrm{m}^{2}\left(\mathrm{WHO}^{26}\right)$. Results:In all the Brazilian states, the overweight prevalence was larger than the one of obesity, in spite of the increment speed to have been higher for obesity. To the prevalece of the beginning to be compared (1980) with the one of the end of the study (2000), it was observed, in Brazil, a reason of prevalência of 2,58 for overweight and of 8,18 for obesity. The prevalece of overweight and obesity shows ascendig curve, with a secular trend. Conclusion: The information obtained in this study points to the necessity of concrete actions to prevent chronic disease in Brazilians young adults.
\end{abstract}

\section{Resumen}

El objetivo de este estudio fué determinar el predominio y la tendencia temporal de sobrepeso / obesidad en brasileños de adolescentes del sexo masculino. Métodos: Se utilizó como fuente de información el banco de datos del Ejército brasileño. Esos datos eran reunido entre 1980 a 2000, y consiste en 4.031.297 adolescentes entre 17 y 19 años de edad. Se realizaron cinco cortes transversales y se cuantificó la prevalencia de sobrepeso/obesidad. Fué considerado sobrepeso el índice de masa corporal (IMC) entre 25 y 29,9 $\mathrm{Kg} / \mathrm{m} 2$, y la obesidad con el mismo índice IMC $>30 \mathrm{Kg} /$ m2(OMS26). En todos los estados brasileños, el predominio de sobrepeso fué mayor que el de la obesidad, a pesar de la velocidad de incremento haber sido mayor para obesidad. La prevalência del principio ser comparado (1980) con el uno del extremo del estudio (2000), fue observado, en Brasil, una razón de prevalência de 2,58 para sobrepeso y de 8,18 para obesidad. Los predominios de sobrepeso/obesidad muestran curvas ascendentes con tendencia temporal en crecimiento. Conclusión: La informaciones obtenidas en este estudio indican la necesidad de acciones concretas para la prevención de enfermedades crónicas en adultos jóvenes brasileños.

Keywords: Overweight. Obesity. Nutritional Transition. Palabras clave: Sobrepeso. Obesidad. Transición Epidemiology. Teen Health.

Nutricional. Epidemiología. Salud del adolescente. 


\section{INTRODUÇÃO}

No Brasil, nas últimas décadas do século XX, verificam-se mudanças no foco das pesquisas realizadas sobre morbimortalidade. Essas apontam a crescente importância da obesidade como tema de investigações científicas. Devido a isso, tem sido acumulado um relativo conhecimento sobre os padrões de determinação e de distribuição da obesidade dentro do contexto da transição nutricional no país. Por outro lado, existe a necessidade de se ampliar o conhecimento no campo da prevenção e da intervenção em populações.

Vários autores ${ }^{1-5}$ afirmam que a situação nutricional em diferentes países vem rapidamente se alterando e que aspectos singulares da transição nutricional são encontrados em virtude das grandes mudanças no estilo de vida, nos padrões de dieta e, conseqüentemente, nas condições de saúde das populações. Essa tendência, no entanto, nunca tinha atingido proporções epidêmicas como atualmente é registrado.

A Transição Nutricional refere-se a mudanças, ao longo dos anos, nos padrões nutricionais que resultam de modificações na estrutura da dieta dos indivíduos, como conseqüência de transformações econômicas, sociais, demográficas e sanitárias ${ }^{6-9}$.

São muitas as causas da obesidade, no entanto são fatores comuns: o declínio no dispêndio energético; a utilização de dietas ricas em gordura (particularmente as de origem animal); o aumento do consumo de açúcar e alimentos industrializados; e a redução do consumo de alimentos ricos em carboidratos complexos e fibras. ${ }^{10-13}$

Dessa forma, a obesidade constitui um dos grandes problemas de saúde pública devido à alta prevalência na infância, à persistência ao longo da vida e às comorbidades associadas. ${ }^{14-16}$

0 incremento da obesidade nos Estados Unidos nos últimos trinta anos foi significativamente elevado. Estudos do Centers for Disease Control mostram que $60 \%$ de adultos americanos têm sobrepeso (CDC, 2003). Essa significativa ascensão ocorreu especialmente nas crianças, nas quais o sobrepeso praticamente dobrou na faixa etária de 6 a 11 anos de idade e triplicou nos adolescentes de 12 a 19 anos de idade ${ }^{17}$.

No Brasil, são ainda escassos e esporádicos os estudos abrangentes de base populacional cujos enfoques sejam prioritariamente os problemas nutricionais. Devemos, porém, destacar o Estudo Nacional sobre Despesas Familiar (ENDEF), realizado entre os anos de 1974 e 1975; a Pesquisa Nacional sobre Saúde e Nutrição (PNSN), de 1989; e a Pesquisa sobre Padrões de Vida (PPV), desenvolvida em 199718.
Com base nesses dois primeiros grandes estudos transversais de abrangência nacional, realizados em 1974 e em 1989, Monteiro et al. (1995b)6, Monteiro e Conde (1999)12 documentam o processo de transição nutricional no Brasil, constatando significativa queda da desnutrição e uma rápida ascensão de prevalência da obesidade. Para Monteiro ${ }^{7}$, a obesidade, nos últimos anos, representa problema nutricional de maior ascensão entre a população adulta brasileira.

De acordo com Kac e Velásquez-Meléndez ${ }^{18}$, é incontestável que o Brasil e diversos países da América Latina estão experimentando, nos últimos vinte anos, uma rápida transição demográfica, epidemiológica e nutricional. As características e os estágios de desenvolvimento da transição diferem nos vários países da América Latina. Um fenômeno, contudo, chama a atenção dos investigadores: o marcante aumento da prevalência de obesidade nos diversos subgrupos populacionais em quase todos os países latino-americanos.

Verifica-se que há uma tendência ao aumento das prevalências de sobrepeso e de obesidade, tanto nos países desenvolvidos quanto naqueles que estão em desenvolvimento. 0 comportamento dessa tendência difere de um país para o outro, e, num mesmo país, e também não é o mesmo em todas as regiões, uma vez que está relacionado às condições socioeconômicas e culturais ${ }^{10,15}$.

Sichieri et al..$^{19}$ chamam atenção para o fato de que, no Brasil, ainda não ocorreu uma verdadeira transição epidemiológica e nutricional, que se caracterizaria pelo quase desaparecimento das doenças por carências e sua substituição por doenças como a obesidade. Entretanto, nesse processo, a transição nutricional já mostra um rápido declínio da maior parte das enfermidades por carências e a ascensão vertiginosa da obesidade e das diversas enfermidades que se associam a ela.

Para Oliveira et al.20 o ganho de peso populacional verificado nas últimas décadas do século XX, no Brasil, parece ser explicado por fatores globais relativos ao consumo de alimentos. Os autores questionam se essas mudanças poderiam ser explicadas por hábitos alimentares incorporados tradicionalmente pela população, por falta de orientação alimentar adequada e, em grande parte, pelo consumo de alimentos hipercalóricos (óleos, açúcares, etc.), por serem mais baratos, e, portanto, mais acessíveis. Também registram que há evidências de que o gasto energético médio sofreu algum grau de redução em decorrência de alterações na forma de viver de boa parte da população brasileira, que tem tido uma vida cada vez mais sedentária.

Segundo OPS8, Monteiro ${ }^{11}$, no Brasil, entre os anos 1960 e 1990, ocorreram grandes mudanças 
econômicas e demográficas. Nesse período, a população urbana aumentou de $45 \%$ para $75 \%$, o ingresso nacional mais que triplicou, e a participação do setor agropecuário na economia baixou de 17,8\% para $6,9 \%$. Esses investigadores questionam, porém, se esses fatores podem explicar tais mudanças no comportamento nutricional.

Sichieri19 e Sichieri et al. ${ }^{21}$ afirmam que o meio ambiente é fator crítico na expressão da carga genética determinante de susceptibilidade para este distúrbio. Para outros autores ${ }^{8,13,22,23}$, os determinantes ambientais também são apontados como as principais causas de obesidade, e, do ponto de vista de saúde pública, o desafio consiste em modificar as condiç̃eses do meio, de tal modo que os indivíduos tenham menos probabilidade de se tornarem obesos.

Monteiro $^{7}$ e Monteiro et al. ${ }^{24}$ afirmam que, a partir de certo nível de desenvolvimento, ricos e pobres tenderão a estar igualmente expostos à obesidade. Assim, dependerá da chamada escolha informada dos indivíduos, em relação à dieta e à atividade física. Portanto, o nível de educação e o acesso à informação dos indivíduos são fatores determinantes.

0 objetivo deste estudo é, então, investigar a prevalência e a tendência temporal do sobrepeso e obesidade em adolescentes masculinos nas cinco macrorregiões e em cada um dos estados do Brasil, no período de 1980 a 2000.

\section{METODOLOGIA}

A população estudada foi de 4.031 .297 adolescentes do sexo masculino, na faixa etária de 17 a 19 anos de idade. Utilizamos como fonte de informação o Banco de Dados do alistamento militar do Exército Brasileiro para todos os estados do Brasil. Como o alistamento militar é uma medida obrigatória e inclui a quase totalidade dos adolescentes jovens nos diversos níveis socioeconômicos, acreditamos ser essa uma amostra uniforme e abrangente.

Realizamos cinco cortes transversais (a cada cinco anos) e quantificamos a prevalência anual do sobrepeso e da obesidade por estado, por região geográfica e em todo o país. A partir das informações seriadas, verificamos a indicação de tendência. De acordo com Pereira ${ }^{25}$, o estudo de série temporal traz um diagnóstico dinâmico da ocorrência de um evento na população, informando a evolução dos riscos a que as pessoas estão ou estiveram sujeitas.

Consideramos como sobrepeso o Índice de Massa Corporal (IMC) entre 25 e 29,9 Kg/m2, e obesidade, o Índice de Massa Corporal (IMC) igual ou maior que $30,0 \mathrm{Kg} / \mathrm{m}^{26}$.
Levamos em consideração todos os estados do Brasil, distribuídos nas cinco regiões: Norte, Nordeste, Sudeste, Sul e Centro-Oeste.

Em relação ao tempo, tomamos os anos de 1980, 1985, 1990, 1995 e 2000.

\section{Plano de análise:}

Os dados foram processados e analisados com 0 auxílio do software EPI-INFO 2000.

0 CD disponibilizado pelo exército contém informações dos adolescentes inscritos para o serviço militar em todo o Brasil, entre os anos de 1980 e 2000.

A partir das informações disponíveis no Banco Primário de Dados, construímos um banco secundário de dados, seguindo as seguintes etapas: a) selecionamos os conscritos registrados em 1980, 1985, 1990,1995 e 2000; b) agrupamo-los por estado e excluímos os conscritos de outros estados alistados fora de seu local de origem; c) excluímos os dados discrepantes em relação à altura e peso (erros de medida); d) selecionamos os conscritos na faixa etária de 17 a 19 anos de idade; e) selecionamos as variáveis idade, peso e altura; f) convertemos a altura de centímetro para metro; g) criamos uma nova variável: Índice de Massa Corporal (IMC), utilizando a fórmula IMC = Peso $(\mathrm{Kg}) /$ Estatura $\left(\mathrm{m}^{2}\right)$.

Calculamos, então, as prevalências do sobrepeso e da obesidade com os respectivos intervalos de confiança de $95 \%$, nos cinco cortes transversais para - Brasil, para as cinco regiões geográficas e para cada estado separadamente. As prevalências de sobrepeso e de obesidade foram calculadas através do método de proporção, conforme orienta Pereira . E, para testar a associação entre as duas variáveis numéricas, utilizamos a correlação linear de Pearson.

\section{RESULTADOS}

Analisando as prevalências de sobrepeso e de obesidade na faixa etária de 17 a 19 anos de idade, verificamos que a curva é ascensional, o que mostra que a tendência temporal nos últimos vinte anos é crescente no Brasil, com uma razão de prevalência de 2,58 para sobrepeso e de 8,18 para obesidade, quando comparada a prevalência inicial (1980) com a prevalência no final do período estudado (2000). Essa tendência epidemiológica foi observada igualmente nas cinco macrorregiões e nos diferentes estados, quando analisados separadamente (Gráficos 1 e 2, Tabelas 1 e 2).

Verificamos que as curvas mostram um declínio do sobrepeso na região Norte (NO) e declínio discreto no Nordeste (NE), de 1980 a 1985, com posterior ascensão 


\section{Gráfico $01:$}

Prevalência (\%) de sobrepeso, em adolescentes do sexo masculino, entre 17 e 19 anos de idade, por região geográfica e ano, no Brasil, 1980 a 2000.

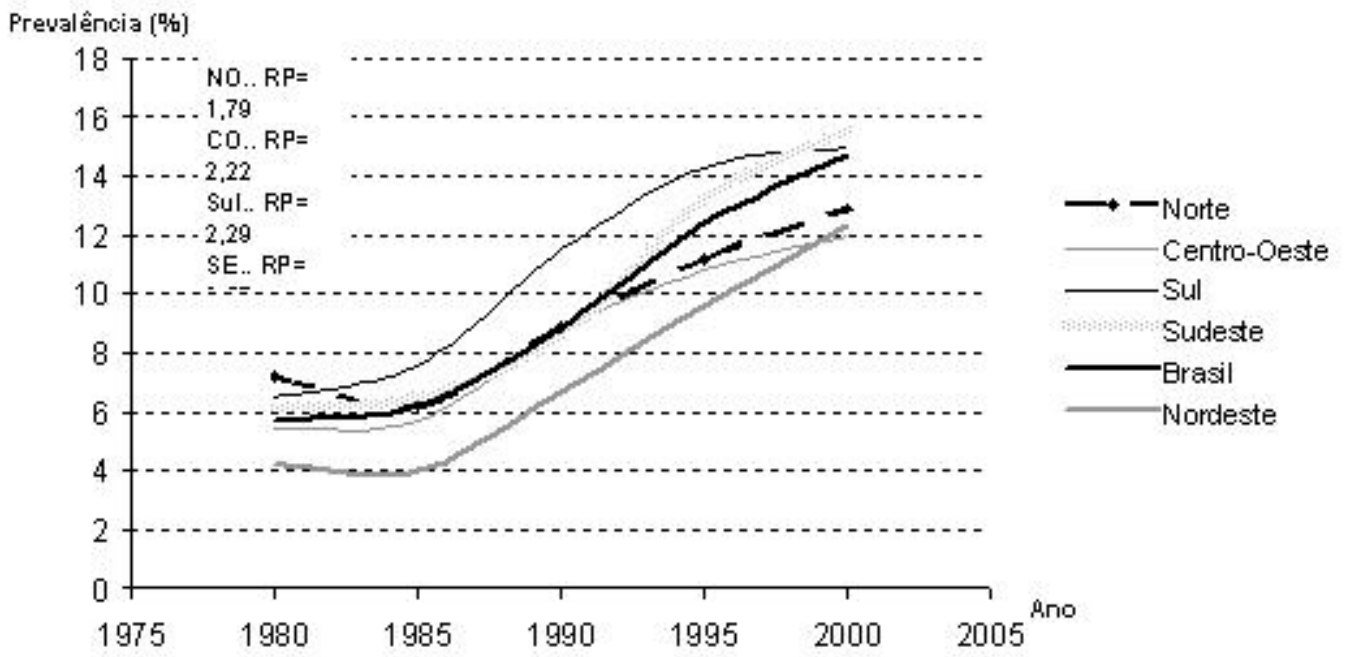

Tabela 1:

Correlação Linear de Pearson para prevalência do sobrepeso em adolescente masculinos na faixa etária de 17 a 19 anos de idade por região geográfica e ano, no Brasil, 1980 a 2000.

\begin{tabular}{l|c|c|c}
\hline \multirow{2}{*}{ Norte } & Pearson & $\mathrm{a}=$ Inclinação & $\mathrm{b}=$ Intercepção \\
\cline { 2 - 4 } Nordeste & 0,935266571 & 0,328 & $-643,44$ \\
Sudeste & 0,964073407 & 0,436 & $-860,28$ \\
Centro-0este & 0,964367996 & 0,092 & $-182,06$ \\
Sul & 0,925004758 & 0,054 & $-106,80$ \\
Brasil & 0,977054302 & 0,088 & $-174,10$ \\
\hline
\end{tabular}

Tabela 2:

Correlação Linear de Pearson para prevalência de obesidade em adolescente masculinos na faixa etária de 17 a 19 anos de idade por região geográfica e ano, no Brasil, 1980 a 2000.

\begin{tabular}{l|c|c|c}
\hline \multirow{2}{*}{ Norte } & Pearson & $\mathrm{a}=$ Inclinação & $\mathrm{b}=$ Intercepção \\
\cline { 2 - 4 } Nordeste & 0,95 & 0,07 & $-134,74$ \\
Sudeste & 0,92 & 0,07 & $-138,74$ \\
Centro-Oeste & 0,97 & 0,09 & $-182,06$ \\
Sul & 0,93 & 0,05 & $-106,8$ \\
Brasil & 0,98 & 0,09 & $-174,1$ \\
\hline
\end{tabular}

\section{Gráfico 02:}

Prevelência (\%) de obesidade, em adolescente masculinos na faixa etária de 17 a 19 anos de idade por região geográfica e ano, no Brasil, 1980 a 2000.

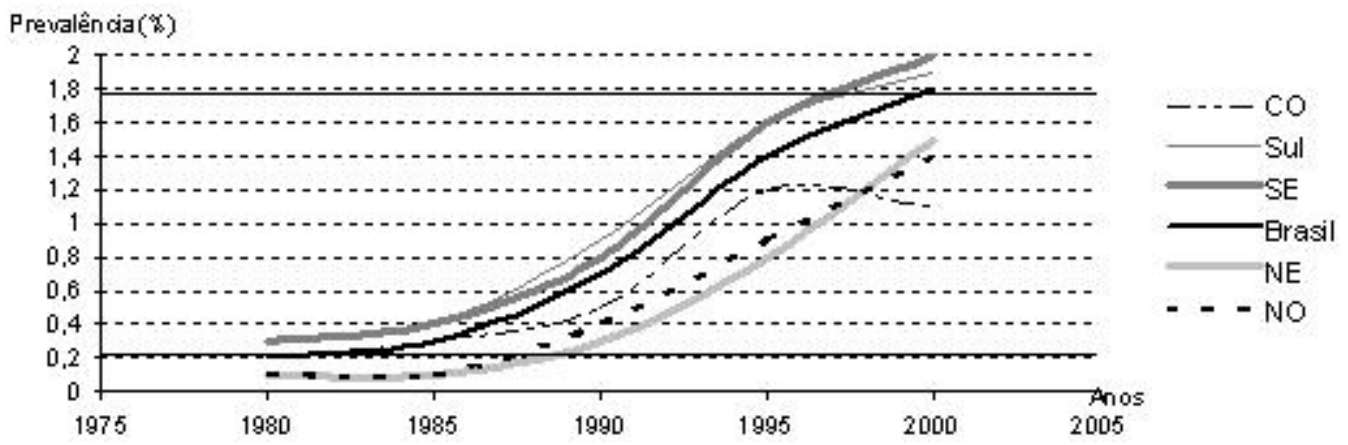


ao longo de todo o período. A velocidade de ganho para o sobrepeso foi maior no Nordeste, com uma razão de prevalência bem acima da do Brasil como um todo, e menor na região Norte (Gráfico 1).

Observamos, ainda, que a curva de obesidade na região Centro-Oeste (CO) foi a única que apresentou uma leve queda na taxa de crescimento no período de 1995 a 2000, contrastando com as demais regiões que sempre estiveram em ascensão (Gráfico 2).

De acordo com os dados da Tabela 3, ao analisar as prevalências de sobrepeso, verificamos que, em todas as regiões, houve uma ascensão desde o início do estudo, exceto nas regiões Norte e Nordeste, que tiveram um declínio de 1980 a 1985, seguido de ascensão até o final do período estudado. Nessa mesma tabela, observamos ainda características que agora passaremos a descrever.

A região Norte apresentou as maiores prevalências de sobrepeso nos anos 1980, 1985 e 1995, porém sua velocidade de ganho foi menor quando comparada com as demais regiões no período estudado. Nessa região, no período de 1980 a 1985, percebemos uma queda acentuada da prevalência de sobrepeso nos estados do Pará, Rondônia e Roraima. Observamos também

Tabela 03:

Prevalência (\%) de sobrepeso, em adolescentes masculinos na faixa etária de 17 a 19 anos de idade por região geográfica e ano, no Brasil, 1980 a 2000.

\begin{tabular}{lccccccc}
\hline LUGAR & 1980 & 1985 & 1990 & 1995 & $\mathbf{2 0 0 0}$ & Média & IC(95\%) \\
\hline Região Norte & $7,2 \%$ & $6,2 \%$ & $8,9 \%$ & $11,2 \%$ & $12,9 \%$ & $9,28 \%$ & 5,$837 ; 12,723$ \\
Região Nordeste & $4,2 \%$ & $4,0 \%$ & $6,7 \%$ & $9,6 \%$ & $12,3 \%$ & $7,36 \%$ & 2,$921 ; 11,799$ \\
Região Sudeste & $6,1 \%$ & $6,5 \%$ & $8,6 \%$ & $13,2 \%$ & $15,6 \%$ & $10,05 \%$ & 4,$768 ; 15,232$ \\
Região Centro-0este & $5,4 \%$ & $5,7 \%$ & $8,8 \%$ & $10,8 \%$ & $12,0 \%$ & $8,54 \%$ & 4,$863 ; 12,217$ \\
Região Sul & $6,5 \%$ & $7,6 \%$ & $11,5 \%$ & $14,3 \%$ & $15,0 \%$ & $10,98 \%$ & 6,$213 ; 15,747$ \\
Brasil & $5,7 \%$ & $6,2 \%$ & $8,9 \%$ & $12,4 \%$ & $14,7 \%$ & $9,58 \%$ & 4,$727 ; 14,433$ \\
\hline
\end{tabular}

Nota: Intervalo de confiança da média das prevalências de sobrepeso (IC 95\%)

uma leve queda nas taxas em Tocantins. Nos demais estados, houve um crescimento progressivo. Em 1985, o estado de Rondônia foi o único do Brasil que mostrou não ter casos de sobrepeso, fato que não se repetiu nos demais anos e locais estudados. Nesse mesmo ano, observamos também uma queda muito acentuada em Roraima. Chamou-nos atenção o fato de, nessa região, o crescimento ter sido muito acentuado desde o início até o final do estudo (1980-2000) nos estados do Acre, Amapá, Pará, Rondônia e Tocantins.

$\mathrm{Na}$ região Nordeste, verificamos que o declínio na prevalência foi menos acentuado que na região Norte de 1980 a 1985 e que essa queda se deve unicamente ao estado da Bahia, visto que os outros estados apresentaram aumento neste período, com destaque para o estado do Piauí que duplicou sua prevalência. Em todos os anos estudados, os estados do Nordeste cresceram acentuadamente, apresentando a maior razão de prevalência para sobrepeso em relação às demais regiões.

No Sudeste (SE), o Estado do Rio de Janeiro foge à tendência no período de 1980 a 1985, apresentando um declínio, enquanto os demais estados apresentam incremento da prevalência. Nessa região, em todo o período analisado, observamos que em todos os estados as taxas de sobrepeso cresceram acentuadamente, vindo a ocupar a posição da segunda maior razão de prevalência em relação às demais macrorregiões do Brasil.

No Centro-Oeste, de 1980 a 1985, destacam-se o Distrito Federal, com acentuada queda, e o Mato Grosso, com significativo crescimento. De modo geral, para todo o período, as taxas de prevalência de sobrepeso cresceram acentuadamente em todos os estados.

Na região Sul, observamos uma maior uniformidade no crescimento em todos os períodos, destacando o estado de Santa Catarina com resultado mais significativo.

Numa análise global, podemos observar que o Sul e Sudeste apresentam prevalências de sobrepeso acima da prevalência média do Brasil em praticamente todos os anos, exceto o Sudeste em 1990.

Ao analisar as prevalências de obesidade no Gráfico 2, verificamos que a razão de prevalência em sua totalidade foi mais alta que a de sobrepeso, e que as curvas de todas as regiões são ascensionais. A região Centro-Oeste apresenta um leve declínio da curva a partir de 1995, diferentemente das demais regiões, onde a curva mostra uma ascensão desde o início do estudo.

Os dados mostraram que o Nordeste teve o maior incremento para sobrepeso e obesidade, alcançando valores maiores que os do Brasil como um todo (Gráficos 1 e 2).

A segunda maior velocidade de incremento para obesidade foi apresentada pela região Norte, e a menor 
velocidade foi constatada na região Centro-0este (Gráfico 2). Ao olhar o Gráfico 1, verificamos que a região Norte apresentou a menor velocidade de ganho para sobrepeso em relação às demais regiões.

Analisando a obesidade (Tabela 4), podemos observar que, na região Norte, a evolução da prevalência da obesidade chama a atenção nos estados de Roraima e Tocantins, no ano 2000, que apresentam taxas superiores às do Brasil, em desacordo com os outros estados da região que apresentam valores inferiores.
Percebemos ainda a inexistência de casos ou de dados de obesidade no início da série no ano de 1980 em três estados do Norte e três do Nordeste; em 1985, em dois estados do Norte e dois do Nordeste; e, em 1990, em um estado do Nordeste. Observamos, portanto, que, ao longo dos anos estudados, a ausência de casos de obesidade foi reduzindo e que, após 1990, tivemos casos de obesidade cada vez mais presentes.

No Nordeste, o estado do Ceará, em 2000, chama atenção por apresentar uma taxa muito alta em

\section{Tabela 04:}

Prevalência (\%) da Obesidade, em adolescente masculinos na faixa etária de 17 a 19 anos de idade por região geográfica e ano, no Brasil, de 1980 a 2000.

\begin{tabular}{l|clllllc}
\hline LUGAR & 1980 & 1985 & 1990 & 1995 & $\mathbf{2 0 0 0}$ & Média & IC(95\%) \\
\hline Região Norte & $0,1 \%$ & $0,1 \%$ & $0,4 \%$ & $0,9 \%$ & $1,4 \%$ & $0,58 \%$ & 0,$000 ; 1,279$ \\
Região Nordeste & $0,1 \%$ & $0,1 \%$ & $0,3 \%$ & $0,8 \%$ & $1,5 \%$ & $0,56 \%$ & 0,$000 ; 1,303$ \\
Região Sudeste & $0,3 \%$ & $0,4 \%$ & $0,8 \%$ & $1,6 \%$ & $2,0 \%$ & $1,02 \%$ & 0,$089 ; 1,951$ \\
Região Centro-Oeste & $0,2 \%$ & $0,3 \%$ & $0,5 \%$ & $1,2 \%$ & $1,1 \%$ & $0,66 \%$ & 0,$087 ; 1,233$ \\
Região Sul & $0,3 \%$ & $0,4 \%$ & $0,9 \%$ & $1,6 \%$ & $1,9 \%$ & $1,02 \%$ & 0,$136 ; 1,904$ \\
Brasil & $0,2 \%$ & $0,3 \%$ & $0,7 \%$ & $1,4 \%$ & $1,8 \%$ & $0,88 \%$ & 0,$013 ; 1,747$ \\
\hline
\end{tabular}

Nota: Intervalo de confiança da média das prevalências de Obesidade (IC 95\%).

relação à de outros estados e por apresentar valor superior à prevalência do Brasil. Na região Sudeste, em todos os anos, observamos taxas superiores às apresentadas no Brasil. Nessa região, no estado de São Paulo aparecem maiores prevalências em relação aos outros estados da região até 1995, exceto no ano 2000, quando é observada uma prevalência inferior à do Rio de Janeiro e do Espírito Santo, mantendo seus valores maiores que a taxa do Brasil em todos os anos.

Na região Centro-Oeste, no Distrito Federal, ocorre um declínio no início e no final do estudo, sendo Brasília uma das responsáveis pelo declínio da curva em 1995, junto com o estado do Mato Grosso do Sul.

Na região Sul, observamos, assim como no Sudeste em todos os anos, que as prevalências são superiores às apresentadas pelo conjunto do país. 0 estado do Rio Grande do Sul se destaca com as maiores prevalências em todos os anos em relação aos demais estados desta região.

Observamos também que, em todos os períodos estudados e na maioria das regiões do Brasil, existe uma forte correlação linear. Exceção se faz ao CO, que mostra uma fraca correlação linear nesse período.

\section{Discussão:}

Os valores absolutos de obesidade foram menores que os de sobrepeso, porém os incrementos foram maiores para a obesidade. Isso pode ser demonstrado tendo em vista que, em duas décadas, o número de jovens obesos do sexo masculino, no Brasil, aumentou em oito vezes. Por outro lado, o número de adolescentes com sobrepeso aumentou duas vezes e meia. Entre os dados apresentados, merece destaque especial o fato de que os valores absolutos altos de sobrepeso mostram a necessidade de, urgentemente, serem realizadas intervenções para evitar a obesidade futura.

Em seu conjunto, os resultados apresentados, quanto à prevalência e à tendência secular do sobrepeso e da obesidade em adolescentes masculinos, não deixam qualquer dúvida sobre a atual importância do problema e sobre seu enorme potencial de expansão, tanto nos estados mais desenvolvidos, como nos menos desenvolvidos, do ponto de vista socioeconômico.

Os achados desse estudo estão em conformidade com os de diversos autores. Halpern27 e Mondini e Monteito28, em inquéritos nutricionais realizados no Brasil, nas três últimas décadas do século XX, verificaram a existência de uma tendência clara no aumento do número de obesos em ambos os sexos. Para esses estudiosos, os dados mostram que, entre 1974 e 1989, a proporção de brasileiros com excesso de peso aumentou de $21 \%$ para $32 \%$. O Sul do país apresenta as maiores prevalências, sendo essas semelhantes e até mesmo superiores às de países desenvolvidos. A evolução da ocorrência nesse mesmo período, em relação ao sexo, dobrou entre os homens (de 
2,4\% para 4,8\%), e, na população feminina, o aumento da obesidade também foi significativo (de $7 \%$ para 12\%). № período de 1975-1989, a evolução da obesidade foi ascendente e relativamente uniforme no Nordeste e Sudeste para homens e mulheres em todas as faixas etárias.

Para os adultos, o aumento da prevalência foi de cerca $90 \%$ para homens. No período de 1989-1997, o aumento foi mais intenso no Nordeste. Na região Sudeste, houve dedínio na prevalência para os estratos de média e alta renda.

É importante ressaltar que não encontramos, na revisão bibliográfica, estudos abrangentes para a população de adolescentes brasileiros. Um estudo da Organização Panamericana de Saúde (2000) revela que aproximadamente $6 \%$ da população brasileira apresentam obesidade ( $1,5 \%$ na região Nordeste e $4,2 \%$ na região Sul). No presente estudo, verificamos que, na população de adolescentes em todo Brasil, no ano de 2000 , a prevalência global de sobrepeso foi de $14,7 \%$, com prevalência de $12,3 \%$ na região Nordeste e 15,0\% na região Sul. Em relação à obesidade em 2000 , a prevalência global foi de 1,8 \%, sendo na região Nordeste de $1,5 \%$, e na região Sul, $1,9 \%$.

Considerando os importantes contrastes sociais existentes no país, em que há uma parcela da população excluída de um consumo alimentar adequado, podemos verificar a permanência de baixa estatura resultante da desnutrição imposta no início da vida. Esse fato é bastante freqüente em alguns grupos menos favorecidos. Nesse sentido, preocupanos a hipótese conhecida como teoria de Barker29, segundo a qual um agravo nutricional ocorrido durante um período crítico do crescimento e desenvolvimento poderá ter efeito deletério durante toda a vida, por induzir mecanismos adaptativos que, na idade adulta, tornarão tais indivíduos especialmente susceptíveis à obesidade. Questionamos, então, se essa é uma das explicações adequadas para os resultados apresentados neste artigo, ou se esses retratam uma outra realidade a partir de outros determinantes básicos explicativos da elevada prevalência de sobrepeso e obesidade verificados.

Também, em nosso estudo, no ano de 1985, observamos uma queda acentuada no estado de Roraima. Questionamos se esse foi de fato um ano atípico, ou se houve problema local com a operação dos dados que venha a justificar essa redução da prevalência naquele ano. Entendemos que as variações observadas nas cinco macrorregiões e entre os estados de uma mesma região necessitam de maiores esclarecimentos que possivelmente serão dados num futuro estudo de correlação ecológica.

\section{CONCLUSÕES}

A prevalência de sobrepeso e obesidade em adolescentes masculinos vem aumentando nas cinco macrorregiões do Brasil e em cada estado separadamente, de 1980 a 2000, e esse aumento, ao longo dos vinte anos estudados, deve ser considerado como um evento sentinela a exigir ações concretas para a prevenção de doenças crônicas em adultos jovens, em futuro próximo, em nível nacional.

\section{Referências}

1. Amigo H. Obesity in Latin American children: situation, diagnóstic criteria and challenges. Cad Saúde Pública 2003; 19(1): 163-70.

2. Brown L, Halweil B, Gardner G. Chronic hunger and obesity epidemic eroding global progress. [on line] 2000 mar; [citado 25 jul 2001]; [aprox.3 telas] Disponível em http:www.worldwatch.org/alerts/ 000304.html.

3. Donald B. About obesity - source: International Obesity Task Force. [on line] 1996 may; [citado 25 jul 2001]; [aprox.12 telas]; Disponível em http://www.obesity.chaire.ulaval.ca/IOTF.htm.

4. The global: epidemic of obesit. [on line] 2001 Sept; [citado 25 jul 2001]; Disponível em http://www.iotf.org/publications/neusletter/spring97.htm.

5. Coitinho DC, Leão MM, Recine E, Sichieri R. Condições nutricionais da população brasileira: adultos e idosos. Brasília (DF): INAN; 1991.

6. Monteiro CA, Mondini L, De Souza AL, Popkin BM. The nutrition transition in Brazil. Eur J Clin Nutr 1995 Feb; 49(2):105-13.

7. Monteiro CA. Epidemiologia da obesidade. In: Lemos MZ. Obesidade. São Paulo (SP): Ed Lab Roche; 1998.

8. Organización Panamericana de la Salude-OPAS. La obesidad en la pobreza: un nuevo reto para la salud pública. Washington (USA); 2000.

9. Gutiérrez-Fisac JL, Regidor E, López GarcíaE, Banegas Banegas JR, Rodríguez ArtalejoF. La epidemia de obesidad y sus factores relacionados: el caso de Espana. Cad Saúde Pública 2003; 19(supl 1): 101-10.

10. Brown LR. Obesity epidemic threatens health in exercise-deprived societies. Worldwatch issue alert. [on line] 2000 dec; [citado 25 jul 2001]; [aprox.2 telas]. Disponível em http://www.worldwatch.org/ chairman/issue/001219.html.

11. Monteiro CA. Velhos e novos males da saúde no Brasil: evolução do país e de suas doenças. São Paulo (SP): HUCITEC; 1995.

12. Monteiro CA, Conde WL. A tendência secular da obesidade segundo estratos sociais: nordeste e sudeste do Brasil: 1975-1989-1997. Arq Bras Endocrinol Metab 1999; 43(3): 186-94.

13. Escrivão MA, Oliveira FLC, Taddei JAAC, Lopez FA. Obesidade exógena na infância e na adolescência. J Pediat 2000; 76(supl 3): 305-10.

14. Popkin BM. The nutrition transition and its health implications in lower income countries. Public Health Nutr 1998; 1:5-21. 
15. Berriós $X$. Tendencia temporal de los factores de riesgo de enfermedades cronicas: la antesala silenciosa de una epidemia que viene? Rev Med Chile 1997; 125:1405-07.

16. Gallagher $\mathrm{S}$. Caring for the child who is obese: morbility, caregiver safety, environmental accommoctation, and legal concerns. Pediatr Nurs 2005; 31(1): 17-20.

17. Linda LH. Childhood obesity: what can be done to help today's youth? Pediatr Nurs 2005; 31(1):13-6.

18. Kac G, Velásquez-Meléndez GA. Transição nutricional e a epidemiologia da obesidade na América Latina. Cad Saúde Pública 2003; 19(supl 1):4-5.

19. Sichieri R. Epidemiologia da obesidade. Rio de Janeiro(RJ): Ed. UERJ; 1998.

20. Oliveira JED, Cunha SFC, Marchini JS. A desnutrição dos pobres e dos ricos: dados sobre a alimentação no Brasil. São Paulo (SP): Sarvier; 1996.

21. Sichieri R, Castro JFG, Moura AS. Fatores associados ao padrão de consumo alimentar da população brasileira urbana. Cad Saúde Pública 2003; 19(supl 1): 47-53.

22. Martins AM. Aspectos genéticos da obesidade: uma perspectiva histórica. Pediatr Moderna 1993; 29(2): 134-41.

23. World Health Organization. Obesity: preventing and managing the global epidemic. Report of a WHO Consultation on Obesity. Geneva; 1998.

24. Monteiro CA, Conde WL, Castro IRR. A tendência cambiante da relação entre escolaridade e risco de obesidade no Brasil (19751999). Cad Saúde Pública 2003; 19(supl 1): 67-75.
25. Pereira MG. Epidemiologia: teoria e prática. $2^{\mathrm{a}}$ ed. Rio de Janeiro (RJ): Guanabara Koogan; 1999.

26. World Health Organization. Physical status: the use and interpretation of anthropometry. Report of a WHO Expert Committee. Geneva; 1995.

27. Halpern A. A epidemia da obesidade. Arq Bras Endocrinol Metab 1999; 43(3): 175-76.

28. Mondini L, Monteiro CA. Relevância epidemiológica da desnutrição e da obesidade em distintas classes sociais: métodos de estudo e aplicação à população brasileira. Rev Bras Epidemiol 1998; 1(1): 28-39.

29. Alves JGB, Figueira F. Doenças do adulto com raízes na infância. Recife (PE): Bagaço; 1998.

\section{Sobre os Autores}

\section{Vera Lucia de Vasconcelos}

Centro de Pesquisa Aggeu Magalhães (CpqAM / FIOCRUZ), Departamento de Saúde Coletiva.

\section{Tiago Maria Lapa}

Centro de Pesquisa Aggeu Magalhães - FIOCRUZ

\section{Eduardo Freese de Carvalho}

Centro de Pesquisa Aggeu Magalhães - FIOCRUZ 